\title{
What would Schrödinger's cat see?
}

Quantum states of light could be a better probe for materials than classical states, but they are hard to generate in the laboratory. A scheme that combines large amounts of data with sophisticated theoretical analysis gets around this limitation.

\section{Carlo Piermarocchi}

aser spectroscopy is a powerful tool for investigating materials. The technique has been used for decades to unravel the complex interactions of electrons and holes in semiconductors. Laser spectroscopy has shown, for example, that electrons and holes form hydrogen-like states known as excitons, which bunch up in biexcitons - the equivalent of biatomic hydrogen molecules and accumulate in electron-hole droplets ${ }^{1}$. Such studies are often carried out in pumpprobe experiments, where a picosecond laser pulse probes the optical absorption after a pump pulse has excited the material. The differential absorption of the probe with and without pumping gives insight into the manybody interactions between carriers.

Lasers thus induce and probe interesting phenomena. In themselves, however, the light sources in these experiments are rather uninteresting. Ideally, a laser gives radiation in a coherent state, with dynamics identical to that of a classical harmonic oscillator. What then if differential absorption experiments were carried out using states of light with properties that are inherently quantum in nature? Writing in Nature Physics,

Mackillo Kira and colleagues ${ }^{2}$ argue that such quantum spectroscopy could probe manybody effects that would be difficult to see with classical spectroscopy. There are many possible states of light with inherently non-classical properties, but Kira et al. focus on a particular class of states that conceptually embody the Schrödinger's cat paradox. These 'cat states' are the quantum superposition of two coherent states symmetrically displaced with respect to a given coherent state. By changing the phase of the displacement, states with different quantum statistics can be obtained. Kira et al. call this process 'stretching' or 'squeezing' cats, depending on whether their quantum fluctuations end up being larger or smaller than for the corresponding coherent state.

In spite of the potential advantages of quantum spectroscopy, experimental realizations are missing, mainly because we do not know how to prepare states of light in arbitrary quantum states. Only a few of all the possible non-classical states of light are routinely generated in the laboratory. The best-known examples are squeezed states, which can be generated using nonlinear methods such as parametric down-conversion ${ }^{3}$. But there are no straightforward experimental procedures for preparing cat states of light.

There is a way around this practical limitation though. Kira and colleagues ${ }^{2}$ have found that the physical realization of a quantum source of light is not necessary. Quantum mechanics is a linear theory, and differential absorption measurements from a source with arbitrary quantum properties can be emulated as a convolution of optical responses obtained using classical sources with an ad hoc quasi-probability distribution. These quasi-probability distributions were introduced, in 1963, independently by Roy Glauber ${ }^{4}$ and George Sudarshan ${ }^{5}$ to demonstrate that any arbitrary quantum state of light has an equivalent integral representation in terms of classical states. Therefore, measurements performed with standard lasers can be processed and used to calculate the response of a virtual quantumspectroscopy experiment, provided the quasi-probability-distribution function is known. Such functions can be negative and non-analytic - this is why we speak of 'quasi-probability' distributions - and the convolution might become difficult to calculate. Moreover, performing the convolution requires information on all possible classical measurements without errors. To make this approach work in practice, Kira et al. have developed a clusterexpansion transformation ${ }^{6}$ that gives a controlled approximation based on a discrete subset of all possible classical measurements.

Kira and colleagues ${ }^{2}$ tested their scheme by performing virtual quantum spectroscopy with cat states in a quantum well, using a large number of classical spectra taken with different values of the field amplitude. It transpires that cat states are indeed better than classical ones at revealing some subtle signatures of many-body correlations. For instance, a stretching cat showed signatures of a bound electron-hole complex larger than a biexciton. Such a state would not be easily detectable by classical spectroscopy.
Many-body interactions in semiconductors are usually studied by performing experiments and by comparing the results with predictions from fundamental theory and computations. In these calculations, physically distinct effects can be 'switched on and off' to gain a fuller understanding. The scheme proposed by Kira et al. ${ }^{2}$ introduces a qualitatively different approach, based on the idea of 'data-intensive discovery'. Recent progress in handling and analysing large amounts of experimental data has led to new ways of producing knowledge in many fields of science. This rapidly expanding approach has been dubbed the 'fourth paradigm of science ${ }^{7}$, adding to the paradigms based on empiricism, theoretical models and computer simulations. In principle, large databases with spectra of materials could be made publicly available, and theorists could analyse them using convolutions corresponding to different quantum sources to enhance and discover many-body features.

Will this data-intensive discovery approach replace the work of theorists? Probably not. Even if many-body features might become more transparent using quantum spectroscopy, the approach does not tell us which many-body states are actually formed in the system, and traditional theoretical and computational methods will remain essential to interpret optical spectroscopy.

Carlo Piermarocchi is in the Department of Physics and Astronomy, Michigan State University, 4263 Biomedical and Physical Sciences Building, East Lansing, Michigan 48824-2320, USA.

e-mail:piermaro@msu.edu

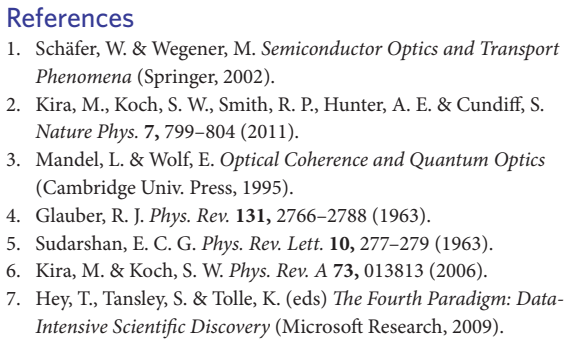

Published online: 18 September 2011 